\title{
Due racconti inediti di Renato Fucini
}

\section{Leonard G. Sbrocchi}

Negli anni in cui Firenze era capitale d'Italia (1865-70), frequentando i vari caffè e le varie farmacie della città - luoghi di ritrovo degli intellettuali dell'epoca, - il giovane aiutante ingegnere Renato Fucini (1843-1921) si fece conoscere ed apprezzare come spirito arguto e brillante per i suoi sonetti dialogati in vernacolo pisano. Servendosi del sonetto come satira, commento (triste o gaio), osservazione psicologica, o come descrizione, il Fucini mostra le molteplici facce del popolino di Pisa. L'angolo visuale dal quale egli proietta questa società è dal suo proprio interno: Neri il personaggio principale che se ne fa interprete è popolano anche lui.

L'acutezza dell'osservazione e la capacità espressiva che da essi emana non sfuggirono a Pasquale Villari il quale, in seguito, lo "arruolò" al suo servizio inviandolo nel 1877 a Napoli col preciso compito di fargli un rapporto dettagliato ${ }^{1}$ sulle condizioni sociali in cui languivano gli abitanti dei bassi della città partenopea. Il risultato di questo viaggio fu il volume Napoli a occhio nudo, una serie di nove lettere con cui il Fucini alternando e contrastando descrizioni della bellezza del paesaggio di Napoli e dintorni con descrizioni estremamente realistiche da parere "allucinanti" del misero stato in cui vivevano i quattro quinti della popolazione di questa città, crea una delle più forti denunce sociali che siano mai state fatte in letteratura italiana. Non è una coincidenza che Giustino Fortunato ${ }^{2}$ nel 1913 lo ristampasse come primo volume della Serie La Questione Meridionale - questione che, purtroppo, a cento anni di distanza non è stata ancora risolta.

Tuttavia l'opera che rese il Fucini uno degli scrittori più rappresentativi della Toscana della seconda metà dell'Ottocento fu Le Veglie di Neri (1882) che porta come sottotitolo "paesi e figure della campagna toscana." Il successo delle Veglie fu consacrato anche dal fatto che dal 1898 al 1949 circa, fu usato come libro di testo nella maggior parte delle scuole italiane. Alle Veglie fece seguito All'aria aperta (1897) anch'esso una collezione di racconti che presentano diversi aspetti della campagna toscana. Ai contemporanei del Fucini ed ai critici in generale questi racconti piacquero per la purezza ed immediatezza della lingua parlata, per la caratterizzazione dei personaggi, e perché erano dei bozzetti a sé stanti, pieni 
di colore locale e pertanto fedeli rappresentazioni (umoristiche o sentimentali) degli abitanti della campagna toscana.

Dopo la morte del Fucini, il prof. Guido Biagi, l'amico di famiglia che ne ordinò le carte, pubblicò due racconti: Nonno Damiano, subito incorporato alle Veglie, e La maestrina ${ }^{3}$ in volume a sé, e in seguito anche incorporato alle Veglie. Perché il Biagi, che pur dovette conoscere questi due racconti che ora noi presentiamo e che certamente posseggono le stesse caratteristiche della maggior parte di quelli contenuti in All'aria aperta, non li pubblicò? La risposta a tale domanda potrebbe essere: non li considerò dello stesso calibro degli altri due; oppure: in confronto La maestrina è un racconto molto lungo e contiene una denuncia sociale fortemente marcata. Inoltre ambedue i racconti hanno molto pathos ed una carica umana assai accentuata, e per questo, forse, dal Biagi considerati racconti "seri." " Quel che alla critica in genere e al Biagi in particolare passò inosservato, forse perché contenuto in un piccolo taccuino di appunti, ${ }^{5}$ è la visione globale della società della campagna toscana che il Fucini si era proposto di presentarci attraverso una serie di quadri che sono appunto i "paesi e figure della campagna toscana."

Dopo l'esperienza napoletana, il Fucini, mettendo a profitto la spigliatezza del dialogo impiegato nei Sonetti e la prosa di Napoli a occhio nudo trovò che la forma più efficace di presentare le condizioni sociali di questi abitanti era appunto il racconto. Questo gli permetteva di mettere a fuoco, in parte o nella loro interezza, a volte dei "paesi," a volte delle "figure," cercando sempre però di cogliere il movente della loro attività, il loro modo di vedere se stessi e gli altri, gli effetti che la società esterna ha su di loro, ed in genere il loro modo di agire. Senza addentrarci in un'analisi dettagliata della novellistica fuciniana, ${ }^{6}$ accenneremo brevemente ai caratteri di questo mondo. Per il Fucini gli abitanti del contado toscano e soprattutto delle Maremme vivono "felici" nel loro ambiente mefitico contentandosi di sbarcare il lunario giorno per giorno perché accettano la loro sorte e trovano soddisfazione in piccole cose quali la caccia e la pesca. Agiscono o per raggiungere il proprio utile o per ignoranza; mescolano religione e superstizione e tuttavia credono in Dio e si rivolgono a Lui in estremo bisogno. I rapporti tra padroni e contadini sono anche basati sul proprio tornaconto, se questo viene a mancare per una ragione o un'altra il contadino perde il lavoro, e l'operaio, in genere è guardato come un animale da sfruttare (Menico). La politica nazionale non li tocca ma essa può avere degli effetti deleteri su di loro (Fiorella). A volte questi abitanti sono anche capaci di azioni generose (Tigrino). La denuncia sociale qui non è né così marcate né così aperta come in 
Napoli a occhio nudo, forse perché i contadini stessi non sempre si ribellano allo status quo.

Ora, nel dare alla luce questi due racconti si è tenuto conto del fatto che, ci sembra, essi contengano degli aspetti di questa veduta d'insieme che non conoscevamo prima. Vero è che il raggiungimento del proprio utile resta il movente principale delle azioni dei personaggi, soprattutto nel primo racconto, nel secondo però esso è altro. Anche la caratterizzazione delle "figure" ha qui un'angolazione nuova. Per esempio, nella sua narrativa il Fucini ci ha dato diverse figure di preti: dal maestro incapace (Il Signor Cappellano) che somministrava la cultura al suono di una canna sulla testa degli studenti, al goloso (Scampagnata), al cacciatore (Fra $i$ due litiganti ...), ma mai uno come Don Remigio dell'omonimo racconto. Don Remigio è ben visto dai contadini non perché sia un prete modello, ma perché, in un certo senso, è uno di loro e in lui essi vedono il raggiungimento del proprio interesse. I superiori non gli avrebbero mai dato una semplice cappellania e tanto meno una parrocchia se i contadini non si fossero ribellati. E Don Remigio approfitta della loro ignoranza e superstizione, come pure della sua conoscenza dell'animo umano, per raggiungere il proprio utile: ottenere una parrochia e con essa il benessere economico. L'angolazione nuova qui consiste nella psicologia di Don Remigio e nel fatto che è la prima volta che i contadini non si ribellano né accusano un prete al contrario lo difendono.

Nel Consiglier Ciabatta più che la patetica figura del consigliere è quella del medico in rapporto ai membri della banda del villaggio che interessa. Diversi medici compaiono nell' opera del Fucini, tuttavia essi sono quasi sempre presentati di scorcio: incisivamente (Dolci ricordi), ove vediamo il medico prima attraverso gli occhi e le apprensioni del figlio, poi attraverso i panni presso il focolare, e infine nel quadro finale che resta indelebilmente impresso nella nostra memoria:

" - Prendi ... Ora è roba tua ...Ma prima di spenderli ...guardami! - e mi fulminò con un'occhiata fiera e malinconica. - Prima di spenderli, ricordati come tuo padre li guadagna.

Una spronata, uno sfaglio e si allontanò a capo basso nel buio, tra la neve e il vento che turbinava."

cauti (Scampagnata) ove mentre tutti sono in chiesa il dottore accenna al personaggio narratore quanto sia difficile la sua vita in quella condotta; o ancora in procinto di perdere questa condotta (Passaggio memorabile) a causa della malevolenza e dell'ignoranza delle giunte comunali. In questo racconto però ne vediamo uno in primo piano che soffre le consequenze del buon esercizio della sua 
professione. Infatti i bandisti (e con essi l'intero villaggio), non si curano del fatto che egli ha salvato la vita al povero Ciabatta da lui prima dato per spacciato, anzi proprio per questo lo vilipendiano, lo maltrattano, gliene combinano di tutti i colori, e infine lo costringono a cercarsi un'altra condotta e a lasciare il paese. Il loro ripicco è che essi hanno concertato inutilmente la marcia funebre in onore del consiglier Ciabatta.

Come tutti i racconti umoristici del Fucini anche questi presentano un umorismo che scaturisce da una sottile ironia. Ma è un umorismo triste che fa da commentario alle azioni dei suoi personaggi e ci spinge a riflettere sulla condizione umana in generale - quasi un "mal giocondo" pirandelliano.

Diamo ora il testo integrale dei due racconti il cui manoscritto si trova nella Biblioteca Riccardiana di Firenze N.3971.

\section{DON REMIGIO}

Povero Don Remigio! tanto bravo, tanto buono, tanto disgraziato. Il Vescovo, a parlargli di lui, si turbava subito; a parlargli di qualche parrocchia o magari d'una povera cappellania vacante per darla a lui, diventava una bestia. Chi sa? Ma, nonostante l'inesplicabile avversione dei suoi superiori, Don Remigio era ben visto dai contadini dei dintorni per la gran passione che aveva al gioco della briscola e perché avevano risaputo, non si sa come, che egli possedeva e teneva sotto chiave un santo miracoloso il quale non aspettava che l'occasione per farsi vivo. Questo santo, a detta dei meglio informati, era specialista abilissimo per i miracoli, diciamo cosí, agricoli. Famoso per ammazzare i topi delle carciofaie; inarrivabile per la distruzione delle grillotalpe quando quelle bestiacce invadevano un campo di poponi o di cocomeri. Ma il miracolo per il quale egli poteva sfidare e vincere di sottogamba tutti i suoi colleghi era quello di far venire a colpu la pioggia e il sereno, secondo il bisogno.

Vacò finalmente una parrocchia vicina. Il Vescovo aveva già pronto il prete da mandarvi, e lo mandò. Ma quel malcapitato ebbe a scappare di rincorsa e ringraziare Dio se gli era riuscito di salvare la pelle perché tutti i contadini di quella parrocchia e delle parrocchie vicine, si sollevarono come un sol uomo, e, prima con le buone, poi con le cattive, vollero che a quella cura fosse mandato Don Remigio. Per vincere questa ribellione furono prese le misure più energiche; ma tutto fu inutile, e le autorità ecclesiastiche e governative, per amore dell'ordine, ebbero a piegare la testa e 
inchinarsi a Don Remigio il quale, in compagnia d'un gatto legato dentro a un sacco e d'una serva che portava una sola valigia per tutt'e due, andò trionfante ad occupare quel posto. Il primo miracolo il santo lo aveva già fatto; ma, nonostante, Don Remigio ebbe subito a scrivere a un figurineo di Lucca perché gli mandasse un busto di gesso da potersi presentare all'adorazione dei suoi parrocchiani come il già celebrato autore di portentosi miracoli che avrebbe fatto.

Il santo di gesso venne, e l'occasione per esperimentarne la bravura non si fece aspettare. Una lunga e ostinata siccità venne a minacciare di un grave disastro la campagna. I contadini di tutta la Toscana erano sgomenti e disperati senza sapere a qual santo ricorrere; quelli della parrocchia di Don Remigio erano, invece, tranquilli e contenti più di prima. Non avevano ancora chiesto il miracolo perché Don Remigio aveva loro detto che non bisognava abusare della bontà e della bravura del suo santo, e che fossero venuti da lui solamente in un bisogno estremo. Venuto questo bisogno, un gruppo numeroso di popolani si presentarono alla canonica.

Don Remigio li ricevette festosamente, e, stropicciandosi le mani dalla contentezza, domandò:

- Ci siete tutti?

- Tutti tutti, nossignore. Ci manca Paolino del Sordo, Isidoro del Birindelli, Gianni Vetturale, Pasquale delle Bard . . .

- Basta, basta. Bisogna che andiate subito a chiamare chi manca perché, lo sapete e ve l'ho detto tante volte, se non ci siete tutti e se tutti non siete della stessa opinione è inutile molestarlo, il mio santo non fa miracoli neanche a frustarlo a legnate.

Dopo una mezz'ora c'erano tutti.

- O bravi bravi! - disse Don Remigio. - Cosí va bene. E allora vediamo.

Fece un buon predicozzo sulla féde, parlò del vino ch'era già rincarato, parlò delle barbabietole, delle granaglie le quali, se non fosse piovuto dentro due o tre giorni, si potevan considerare come perdute, e concluse col riconoscere che non c'era tempo da perdere. E poi si venne subito alla votazione.

- Chi alza la mano - esclamò Don Remigio - approva che si chieda subito la pioggia, chi non l'alza lo disapprova.

Dei trentadue presenti, trenta soli alzarono la mano. Due stettero fermi.

- Male, male di molto questa faccenda! - brontolò Don Remigio - Che v'è saltato in testa a voi due? Presto, presto! Sentiamo! -

Il primo dissenziente, grattandosi la zucca, osservò: 
- Se piove dentro tre giorni ... anche dentro la settimana, io Signor priore, son rovinato. Se i contadini voglion l'acqua, padroni. Ma noi lavandai ... Dio del cielo! Oh, la facci baia e non canzoni! C'è da asciugare un bucato che fa paura, e se quelle donne sapessero che anch'io mi son messo d'accordo per far piovere mi mangerebbero vivo. -

Don Remigio gongolava; e volgendosi inviperito all'altro dissenziente, gridò:

- E voi? Sentiamo anche voi! . . Ma dunque non sarà possibile?

.Vergine santissima! La campagna brucia, il tempo scappa ... Sentiamo, ne! -

- Lei deve riflettere, Signor Priore, che d'aria non si campa e che noi vetturali ...si deve figurare che questa settimana ci ho tre spacci da buscare una venticinquina di lire; e tutt'e tre m'hanno fatto dire che se piove non se ne fa nulla ... Dunque lei vede che se io $\mathrm{mi}$ rifiuto e se io ....-

Fu impossibile metterli d'accordo. Il lavandaio e il vetturale piantarono le spalle al muro, e chi vuol Cristo se lo preghi. Si presentarono presto altre bellissime occasioni, ma i risultati furono uguali a quelli della prima. Tantoché il santo di Don Remigio fa la vita del gaudente, col buzzo pieno di miracoli senza poterne buttar fuori neanche uno; e Don Remigio, deplorando la incorregibile caparbietà e le inevitabili divergenze dei cervelli umani, se la gode allegramente, soccorso in ogni occasione dal pollaio, dalla cantina e dalla fede dei suoi popolani.

\section{IL CONSIGLIER CIABATTA}

Sebbene i suoi compaesani gli avessero appioppato l'ignobile soprannome di Ciabatta, egli, che si chiamava Alcibiade Carcassi, godeva la stima e la benevolenza di tutti per la rispettabile casata alla quale apparteneva, per il suo grado di consigliere comunale e, soprattutto, per la protezione che, larga e disinteressata, aveva sempre accordato alla banda musicale del Comune. Non so se fosse cavaliere, ma credo di sí. Il suo fratello, oggi sindaco e presidente del consorzio per le arginature dei fiumi, lui è cavaliere di certo perché ne lessi la nomina sulla gazzetta ufficiale. Di lui, come dicevo, non lo so; ma me ne voglio informare.

Il soprannome di Ciabatta gli venne perché, qualche anno addietro, soffrendo d'un incomodo ne' piedi, fu costretto per qualche mese a non poter portare altra calzatura che le ciabatte. Se ne trovò in seguito tanto bene che non poté quasi piú abbandonare 
quel genere di calzatura. Sui primi tempi cominciò a mettersi le scarpe solamente la domenica, poi per le solennità, poi per la festa del Titolare e per quella del Corpus Domini solamente, e da ultimo, per il Corpus Domini solo perché il proposto messe i piedi al muro e non gli permise in nessun modo di venire a processione con le ciabatte.

Ma in sostanza, dentro a quel soprannome c'era tutta la stima e tutta la simpatia dell'intera popolazione, esemplare, tranne quattro o sei rompicolli, per una popolazione per codardia assolutamente rara e per intelligenza molto comune; perché quelle ciabatte erano agli occhi di tutti un indiscutibile attestato di indipendenza e di democrazia.

E fin qui tutto sarebbe andato bene. Ma quello che non andava tanto bene era la salute del povero Ciabatta. Dopo quella tremenda indigestione che prese quando cantò messa il suo nipote di Gamberaia, non ha avuto più pace. Pareva da principio, che si trattasse di cosa leggera; ma da qualche giorno le cose avevano preso una piega cosí brutta, da mettere seriamente in pensiero. Disappetenza assoluta, vertigini continue e un colore della pelle cosí giallo da non lasciar dubbio su gravissime complicazioni al fegato.

Il medico ne parlò una sera in farmacia, e ne parlò in modo da mettere lo sgomento in tutti quelli che lo ascoltavano perché, secondo lui, tranne il caso di una reazione non punto sperabile da organismi dell'età e del temperamento del povero Ciabatta, non c'era da illudersi sulle condizioni disperate del malato.

Il maestro della banda, che si trovava presente a quei discorsi, era il piú sconcertato di tutti. Nel repertorio del corpo musicale da lui diretto non c'era una marcia funebre!

- E glie l'ho detto, glie l'avrò detto mille volte a quelli zucconi - esclamava il maestro, asciugandosi il sudore della faccia desolata, - mille volte glie l'avrò detto: impariamola, ragazzi, una marcia funebre ... .i casi son tanti . . .non si sa mai.

- Perbacco! - interruppe il medico - gliela faccia imparare ora! Non ho detto mica che la catastrofe possa accadere da oggi a domani! ..

- E, press' a poco, quanto potrà andare in là?

- Se fossi indovino, caro maestro, sarei ricco.

- Ma press'a poco? ...

- Secondo le complicanze che possono sorgere, amico mio; ma è certa che quel malato lí può andare avanti benissimo ... che so io? ...Perché a volte quelle affezioni epatiche ... Insomma domattina lo rivedrò e le saprò dire meglio a che punto sono le 
cose .. Scusi, maestro, quanto tempo le ci vuole per mettersi in ordine? ...

- Per lo meno una ventina di giorni.

- Eallora, lei vada tranquillo; resto garante io ...

- Ma proprio? proprio me l'assicura?

- Lei mi conosce. Conti sulla mia parola ...

Il maestro andò via tutto rasserenato, e la sera stessa fu messo mano alle prove della marcia funebre.

Furono prese, dalla famiglia e dagli amici, tutte le precauzioni, perché il suono degli strumenti non arrivasse alla camera del malato, ma, essendo tanto piccolo il paese e tanto vicina la sala della banda, non fu possibile ottenere lo scopo quantunque i bandisti suonassero a finestre chiuse, sudando come bestie, e fossero soppresse tutte le botte dell'ofleide e della grancassa.

Il povero Ciabatta sentiva tutto e, voltandosi nel letto dalla parte della finestra, diceva, sorridendo malinconicamente: questa la imparano per me!

- No, no, zio.

- No, signor Alcibiade.

- Ma guarda che malinconie ti vai a mettere per la testa! -

Il nipote, gli amici ed il fratello cercavano di ingannarlo, ma il povero Ciabatta non si lasciava ingannare e, con un misto di speranza, d'orgoglio soddisfatto e di rassegnato dolore:

- Questa la imparano per me! -

Una lettera anonima, diretta al Signor Alcibiade Carcassi urgentissima, venne a scoprire tutte le batterie. Pezzo d'assassino! Ma chi sia stato quel birbaccione che, a rischio di far morire un uomo sul colpo ... Dev'essere stato quello! Dev'essere stato quell'altro! Figlio d'un cane!

- Se arrivo a scoprirlo gli mangio un orecchio! - urlò il maestro della banda, vedendosi minacciato della morte di Ciabatta prima del tempo.

I sospetti caddero su molte persone, ma nessuno, com'è naturale, mise gli occhi addosso a quella sola che aveva ragioni e che poteva esser capace di tanta infamia, nessuno pensò a quel figuro di Nazzario che due mesi fa era stato espulso dal corpo musicale perché s'accorsero che tutte le sere di prova, appena spenti i lumi, si metteva in tasca tre o quattro mozziconi di candela.

Ma la lettera produsse l'effetto opposto di quello che tutti si aspettavano. Appena il povero Ciabatta non ebbe più dubbi che la marcia funebre la preparavano proprio per lui, fu come metter l'olio nel lume, fu tanta la sua consolazione fu cosí grande la sua compiacenza che dette in un gran pianto di tenerezza e cominciò subito a migliorare a vista d'occhio. 
Tutti volevano fino, da quel momento, cessare le prove, ma lui non volle; anzi valendosi dei diritti che gli accordava la sua munifica protezione al corpo musicale, impose che non solo si continuassero le prove, ma si continuassero con l'ofleide, con la grancassa e con le finestre aperte.

E cosí fu fatto.

Alla sesta prova egli poté strascinarsi, per sentir meglio, fino a una poltrona accanto alla finestra; e lí, beandosi, stava ad ascoltare con gli occhi umidi di tenerezza, fissi nel cielo stellato.

Che bivio terribile per quell'anima di vecchio fanciullo! Da una parte il trasporto, la banda, il lamento delle campane, il popolo schierato lungo la via, piangente e senza cappello! Dall'altra parte lo spaventoso mistero ...forse il purgatorio ...forse ...! Che orrore che orrore!

Una sincope. Quello che ci sarebbe voluto per lui sarebbe stata una sincope. Ma una di quelle sincopi a scarica fissa come le sveglie, e che lo scatto fosse accaduto appena finiti i discorsi sul feretro e innanzi il primo colpo sui chiodi della cassa.

E pensando a questa sincope il suo viso si irradiava di tanta gioia da sembrare che dagli occhi e dalle bocca gli colasse il miele fonte a fonte.

E i bandisti, uscendo dalla prova, si fermavano sotto la sua finestra a domandargli come stava.

- Va benino, va benino. Grazie giovinotti.

- Ci rallegriamo, Signor Alcibiade. Si riguardi. Buona notte, buon riposo.

E se ne andavano per la strada buia, brontolando sotto voce:

- Figlio d'un cane che pelle dura!

- Eppure guarisce davvero!

- Quello, vedete, dev'esser peggio dei rospi che, anche infilati con una canna, stanno tre giorni a sgambettare, con la pancia al sole!

- O il dottore che dice?

- Lui seguita a dire che non ne può levare le gambe.

- Speriamo! -

Il Consigliere Ciabatta intanto migliorava di giorno in giorno, e i bandisti e i molti loro aderenti tenendo il medico responsabile della bella figura che avevan fatto, provando da due mesi la marcia funebre per ....per . . . per chi?, lo guardavano di traverso, e i più turbolenti non si peritavano a rivolgergli parole aspre e monosillabi minacciosi.

- Se Ciabatta guarisce, il medico la paga!

Ma Ciabatta andava di bene in meglio. Adagio adagio ricominciò a nutrirsi, messe più carne e colore, e gli tornò la forza nelle gambe, 
tanto da cominciare a girellare per l'orto e sul marciapiede davanti a casa, reggendosi a un bastone. Gli fu prescritta la cura del sangue dalla quale ricavò vantaggi sensibilissimi, e dopo otto giorni di Montecatini, ritornò, Dio glielo perdoni, più fresco, più sano e più giovane di dieci anni.

Le prove della banda che, da qualche giorno erano state rallentate, dopo il ritorno di Ciabatta dalla Val di Nievole, furono bruscamente interrotte, e scoppiò finalmente contro il medico il malumore di tutta la popolazione, che fino allora aveva covato minaccioso in silenzio.

I muri delle case si empirono di - morte al medico - di teste di somaro e di corna incrociate col suo nome sotto. Non si salvarono dalla tempesta né sua moglie né i suoi cinque figlioli. Sputi quando passavano, parole sconce, fischi e baiate. Urli, immondizie e sassate nelle finestre la notte. Eppoi penne e vetri nel fieno del cavallo, sfregi al servitore, e finalmente tutti i cavoli dell' orto sbarbati e un gatto marcio in casa dalla finestra.

Vista la mala parata, il medico cercò di un'altra condotta, vi concorse e l'ottenne.

Il giorno che, affastellata tutta la famiglia in una diligenza e i mobili sopra un barroccio, se ne andò dal paese non ebbe né un addio né una stretta di mano, ma risa sgangherate e - buon viaggio - ironici di dentro alle botteghe e di dietro ai muri degli orti.

Il solo che mostrò la sua riconoscenza al medico, fu il povero Ciabatta il quale, informato della partenza del dottore, lo aspettava sulla porta per salutarlo.

\section{University of Ottawa}

\section{NOTE}

1 In proposito vedi il mio saggio: "Carteggio Villari-Fucini," Critica Storica, A.XI, Nuova serie, N.3 (Settembre 1974), 481-90.

2 Giustino Fortunato (1848-1932) di Rionero in Vulture (Potenza), fu deputato al Parlamento (1880) e poi senatore (1908), pubblicò diversi libri sulla questione meridionale. Nel 1877 fece da guida al Fucini durante il suo soggiorno a Napoli. Vedi i miei due scritti: "Carteggio Fortunato-Fucini," Critica Storica, A.X, Nuova serie, N.4 (Dicembre 1973), 684-91, e "Fucini-Fortunato: Napoli a occhio nudo," Critica Storica, A.X111, Nuova serie, N.1 (Marzo 1975), 168-75.

3 Confrontando il testo del Biagi col manoscritto originale per un'edizione critica, ho trovato che il Biagi ha fatto dei "tagli" a volte considerevoli.

4 Questo possiamo rilevarlo anche dalla scelta da lui fatta per un'Antologia degli scritti di Renato Fucini, per le scuole e per le famiglie, con alcune pagine inedite e con note di Guido Biagi (Firenze, 1923).

5 Vedi il capitolo quarto del mio saggio Renato Fucini l'uomo e l'opera (Firenze, 1977).

6 Per un'analisi dettagliata vedi il mio Renato Fucini. 